\title{
Immunohistological detection of Chlamydia pneumoniae in the Alzheimer's disease brain
}

\author{
Christine J Hammond ${ }^{1,3}$, Loretta R Hallock', Raymond J Howanski ${ }^{1}$, Denah M Appelt ${ }^{2,3}$, C Scott Little \\ Brian J Balin ${ }^{1,3^{*}}$
}

\begin{abstract}
Background: Sporadic late-onset Alzheimer's disease (AD) appears to evolve from an interplay between genetic and environmental factors. One environmental factor that continues to be of great interest is that of Chlamydia pneumoniae infection and its association with late-onset disease. Detection of this organism in clinical and autopsy samples has proved challenging using a variety of molecular and histological techniques. Our current investigation utilized immunohistochemistry with a battery of commercially available anti-C. pneumoniae antibodies to determine whether $C$. pneumoniae was present in areas typically associated with AD neuropathology from 5 AD and 5 nonAD control brains.

Results: Immunoreactivity for C. pneumoniae antigens was observed both intracellularly in neurons, neuroglia, endothelial cells, and peri-endothelial cells, and extracellularly in the frontal and temporal cortices of the AD brain with multiple C. pneumoniae-specific antibodies. This immunoreactivity was seen in regions of amyloid deposition as revealed by immunolabeling with two different anti-beta amyloid antibodies. Thioflavin $\mathbf{S}$ staining, overlaid with C. pneumoniae immunolabeling, demonstrated no direct co-localization of the organism and amyloid plaques. Further, the specificity of $C$. pneumoniae labeling of AD brain sections was demonstrated using C. pneumoniae antibodies pre-absorbed against amyloid $\beta$ 1-40 and 1-42 peptides.

Conclusions: Anti-C. pneumoniae antibodies, obtained commercially, identified both typical intracellular and atypical extracellular C. pneumoniae antigens in frontal and temporal cortices of the AD brain. C. pneumoniae, amyloid deposits, and neurofibrillary tangles were present in the same regions of the brain in apposition to one another. Although additional studies are required to conclusively characterize the nature of Chlamydial immunoreactivity in the AD brain, these results further implicate $C$. pneumoniae infection with the pathogenesis of Alzheimer's disease.
\end{abstract}

\section{Background}

Alzheimer's disease (AD) is a progressive neurological disease that affects millions of older individuals. Distinctive pathological hallmarks associated with the disease include tau accumulations forming neuropil threads (NTs) and neurofibrillary tangles (NFTs), and deposits of extracellular amyloid comprising neuritic senile plaques (NSPs). In general, there are two distinct forms of Alzheimer's disease, familial AD and sporadic late-onset AD. The early onset form of the disease, known commonly as

\footnotetext{
* Correspondence: BrianBa@pcom.edu

'Pathology/Microbiology/Immunology and Forensic Medicine Department, Philadelphia College of Osteopathic Medicine, 4170 City Ave, Philadelphia, Pennsylvania, USA

Full list of author information is available at the end of the article
}

familial $\mathrm{AD}$, is caused by dysregulation of many processes due to genetic mutations that lead to the aforementioned pathology. For example, mutations in presenilins 1 and 2 genes and the gene responsible for amyloid- $\beta$ protein precursor $(\mathrm{A} \beta \mathrm{PP})$ result in an increased accumulation of beta-amyloid $(A \beta)$ in the brain. In late-onset sporadic $\mathrm{AD}$, similar pathological accumulations occur, although without the gene mutations seen in familial AD (for review see Duyckaerts 2009) [1].

Most investigations have focused on the extracellular, deposited forms of amyloid in the AD brain. The extracellular accumulations of amyloid in the brain are composed principally of amyloid $\beta \quad 1-40$ and 1-42 and form neuritic senile plaques (NSP) [1,2]. However, intracellular accumulations of amyloid, which may occur prior to

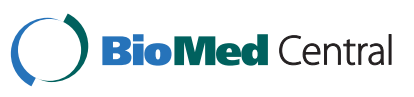


extracellular deposition, also have been demonstrated [3-6]. Further, A $\beta$-derived diffusible ligands (ADDLs), soluble forms of amyloid, have been postulated to be a toxic form of amyloid at the synapses and are not found in typical neuropathological or histopathologic accumulations of amyloid [7]. Since late-onset AD lacks the same mutations seen in familial AD, determination of the cause of amyloid pathology in late-onset $\mathrm{AD}$ remains poorly understood.

The interplay between normal processes and environmental factors, both independently and in concert with other genetic factors, may lead to late-onset AD. In particular, infections as environmental factors may have an impact on the delicate amyloid and tau balance in the brain and lead to the pathology seen in AD. A number of infectious agents have been associated with late-onset AD [8-12]. Our focus has been on the obligate, intracellular bacterium Chlamydia pneumoniae, which has been demonstrated to be highly prevalent in the AD brain $[8,12]$, as well as associated with other systemic and neurological diseases $[13,14]$ including atherosclerosis $[15,16]$, stroke [17], encephalitis [18], and multiple sclerosis [19].

Various cell types found in the brain have been shown to be susceptible to infection by $C$. pneumoniae including endothelia, astroglia, microglia, and neurons [8,12,20-23]. Once inside the cell, $C$. pneumoniae reside in an intracellular inclusion that resists lysosomal fusion and immune recognition. C. pneumoniae developmentally cycle from the infectious elementary body (EBs) to the metabolically active reticulate body (RBs), which divide by binary fission. This obligate intracellular pathogen both interacts with and manipulates the host by gathering energy and nutrients that are required for replication, such as sphingomyelin and cholesterol [24-26]. Chlamydiae also inhibit apoptosis [20,27-29] and release factors such as chlamydial lipopolysaccharide (LPS) and glycolipid protein into both the cell itself and into the surrounding milieu [30]. Further propagation and spread of the organism may follow one or more pathways. The infectious progeny, EBs, may be released upon eventual cell death or by extrusion from the cell in a membrane bound package into the surrounding environment [31]. Thus, C. pneumoniae and/or antigens derived from the organism may be localized both intracellularly and extracellularly at the site of infection.

Due to the chronic nature of AD and the complexity of C. pneumoniae infections, establishing an association with disease pathogenesis has proved difficult. Validating this association relies on a variety of detection methods for the organism. Our current study focuses on the use of immunohistochemistry (IHC) with a battery of commercially available anti-chlamydia antibodies on frontal and temporal cortical sections of human AD brains.
Our data suggest this methodology provides a valuable insight into the interrelationship between infection and AD pathology.

\section{Results}

\section{C. pneumoniae immunolabeling in AD tissue}

All available sections from the frontal and temporal cortices of AD and control brains were immunolabeled with anti-C. pneumoniae antibodies listed in table 1. All AD brains were found to immunolabel with all of the anti-C. pneumoniae antibodies, although the type of labeling profile (eg, intracellular versus extracellular) and extent of label differed. Representative labeling profiles were highlighted in Figure 1. An AD brain immunolabeled with no primary antibody and both an anti-mouse horseradish peroxidase (HRP) conjugated secondary and an anti-mouse alkaline phosphatase (AP) conjugated secondary reacted with both $3,3^{\prime}$-Diaminobenzidine (DAB) and AP red illustrate the absence of non specific immunolabeling in the temporal cortex. The AD frontal cortex labelled with a mouse anti-C. pneumoniae monoclonal antibody (Table 1, \#5) demonstrated both neuropil (Figure 1B) and intracellular (Figure 1C, D) immunolabeling. C. pneumoniae was present in the hippocampus and entorhinal cortex of Alzheimer's disease brain (Figure 2). Representative immunolabeling with an antiC. pneumoniae monoclonal antibody (Table 1, \#5) was observed in the dentate gyrus of the hippocampus indicated by the magenta color (Figure 2A, B). C. pneumoniae immunolabeling also was observed in apparent large neurons within the entorhinal cortex (Figure 2C, D) that also contained intraneuronal lipofuscin accumulations (golden brown, arrowheads). Although the chlamydia immunoreactivity was detected using all anti-chlamydia antibodies in all AD brains, the extent of the label varied with the antibody used and AD case examined. Chlamydia immunolabeling was detected in the frontal and temporal cortices and appeared distributed across all 6 cortical layers. Although the immunolabeling may appear robust in some areas, in general, the intracellular immunoreactivity was observed in less than $1 \%$ of brain cells in

Table 1 Commercially available Chlamydia antibodies

\begin{tabular}{|c|c|c|c|c|}
\hline 1 & $\begin{array}{l}\text { BioDesign, Meridian Life } \\
\text { Sciences }\end{array}$ & $\mathrm{B} 65256 \mathrm{R}$ & Polyclonal & $1: 100$ \\
\hline 2 & $\begin{array}{l}\text { BioDesign, Meridian Life } \\
\text { Sciences }\end{array}$ & C65165M & Monoclonal & $1: 100$ \\
\hline 3 & $\begin{array}{l}\text { BioDesign, Meridian Life } \\
\text { Sciences }\end{array}$ & C65691M & Monoclonal & $1: 100$ \\
\hline 4 & Fitzgerald & $10-C 27$ & Monoclonal & $1: 100$ \\
\hline 5 & GenWay Biotech & $\begin{array}{l}20-902- \\
170121\end{array}$ & Monoclonal & $1: 100$ \\
\hline 6 & GenWay Biotech & $\begin{array}{l}20-272- \\
190984\end{array}$ & Monoclonal & $1: 100$ \\
\hline
\end{tabular}




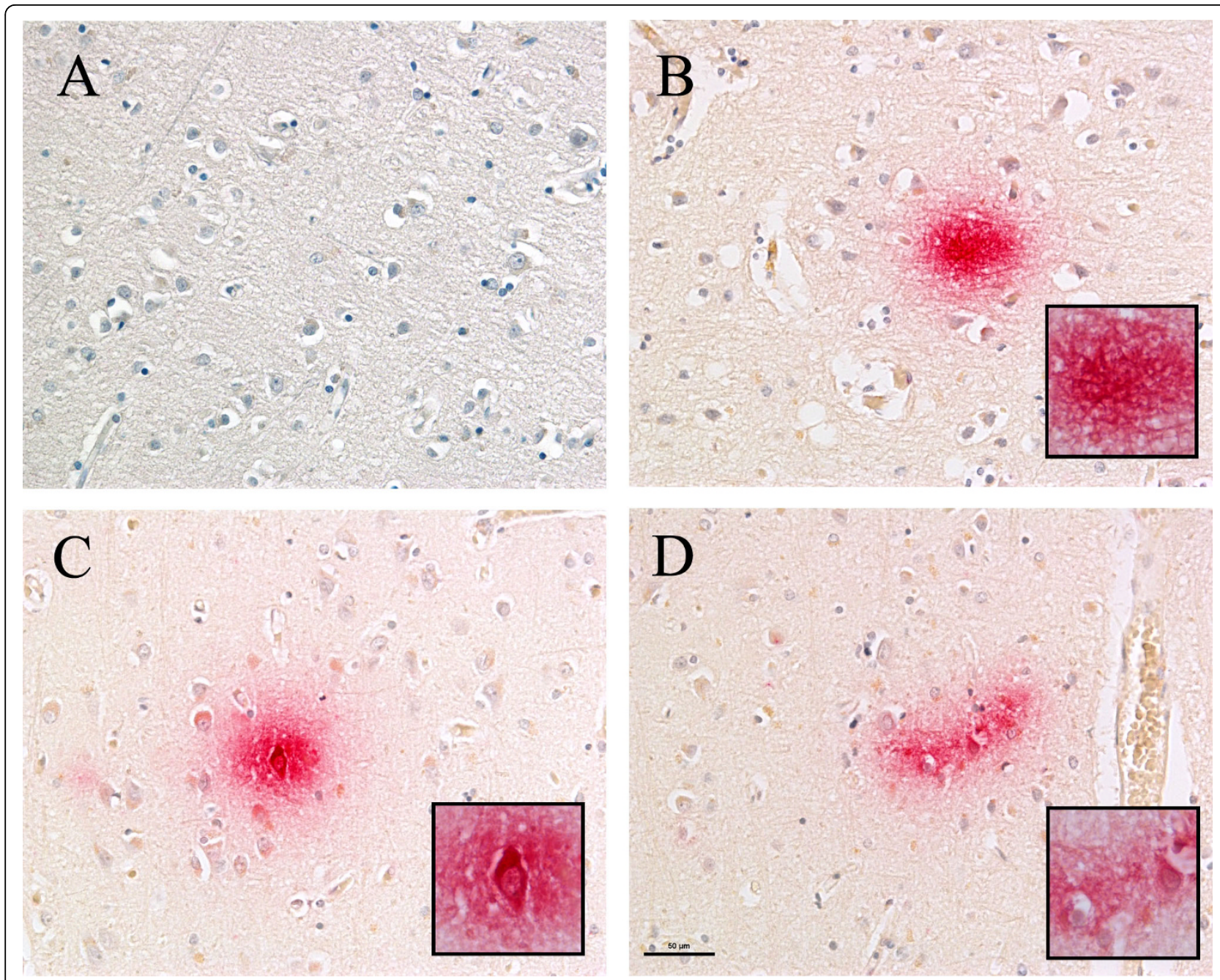

Figure 1 Chlamydia pneumoniae immunoreactivity in AD brain tissue. Alzheimer's disease brain cerebral cortex immunolabeled with a mouse anti-C. pneumoniae monoclonal antibody (Table 1, \#5) demonstrates both intracellular and extracellular immunolabeling as indicated by the magenta color. Panel A illustrates the temporal cortex labeled with no primary antibody and both anti- mouse HRP and anti-mouse AP conjugated secondary antibodies reacted with both DAB and AP red. Panel B illustrates an extracellular labeling pattern and panels $C$ and D illustrate cellular labeling in the frontal cortex. Areas delineated by the boxes are higher magnification images of the immunoreactivity in panels B-D. Size bar $=50 \mu \mathrm{m}$.

these regions. The cell types in the grey matter within the hippocampal formation (Figure 2E) that were labeled intracellularly include neuroglia, large and small neurons, pyramidal neurons, although less commonly, and perivascular cells. Similar cell types showed immunoreactivity across all frontal and temporal cortical layers. This intracellular immunoreactivity was occasionally seen in cells with granulovacuolar degeneration and tangles in these regions. Additionally, the more atypical extracellular chlamydia immunoreactivity was visualized across all cortical layers with approximately $1-2 \%$ of the area in a chlamydia-positive field demonstrating immunoreactivity. This varies widely with different AD cases. Further, the immunoreactivity was seen in the white matter and even the cerebellum although study of this immunolabeling was outside the scope of this report. Minimal immunoreactivity was seen in the 2 of 5 non AD cases in the frontal and temporal regions, not confined to any specific layer; and there was some hippocampal involvement.

Amyloid and C. pneumoniae immunolabeling in AD tissue Amyloid and C. pneumoniae immunoreactivity were detected in sections of the AD frontal (Figure 3A, B) and temporal cortices (Figure $3 \mathrm{C}, \mathrm{D}$ ). Amyloid immunoreactivity was present in dense core mature plaques (brown color) using a rabbit anti-amyloid 1-42 antibody (Sigma) (Figure 3A), and a mouse monoclonal anti-amyloid antibody (4G8, Signet) which also demonstrated 

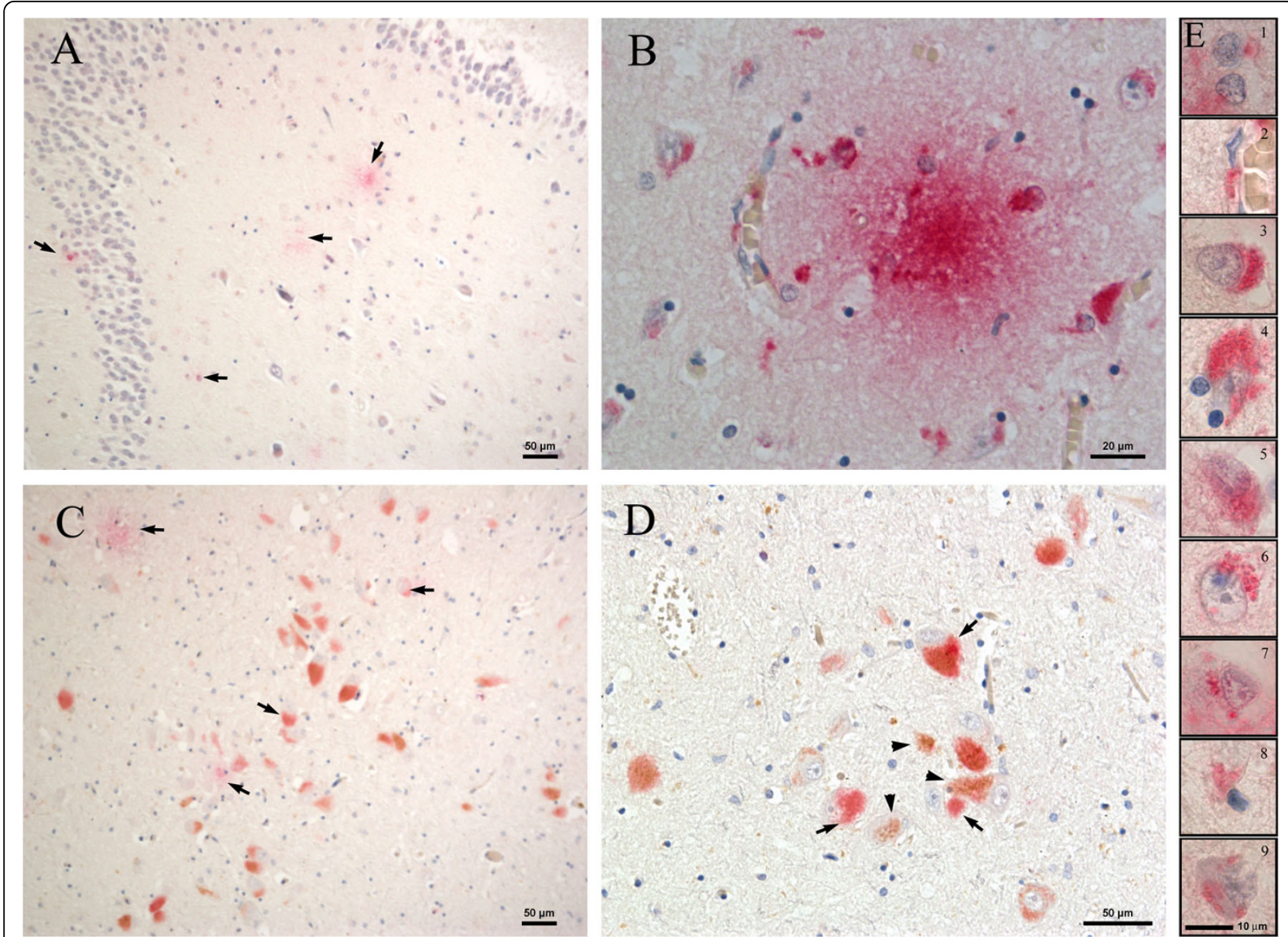

Figure 2 Chlamydia pneumoniae immunoreactivity in hippocampus and entorhinal Cortex. Detection of $\boldsymbol{C}$. pneumoniae antigen in the Alzheimer's disease hippocampus in panels A and B. Representative immunolabeling with mouse anti-C. pneumoniae monoclonal antibody (Table 1, \#5) shows immunopositivity in the dentate gyrus of the hippocampus. Immunolabeling is indicated by the magenta color and is denoted by the arrows in panel A. Extracellular and intracellular $\boldsymbol{C}$. pneumoniae immunolabeling in apparent large neurons is found within the entorhinal cortex, panels $C$ and D. Prominent labeling (magenta) denoted by the arrows in panels $C$ and $D$, is easily distinguished from intraneuronal lipofuscin (golden brown) denoted by the arrowheads (panel D). High magnification images of chlamydial labeling within different cell types in the hippocampal region are illustrated in panel $E$ (1, 8: neuroglia; 2: peri-vascular; 3, 4, 5, 6, 7, 9: neuronal). Note the apparent tangle within the neuron in E9.Size bars: $A, C$, and $D=50 \mu m ; B=20 \mu m ; E=10 \mu m$.

intraneuronal amyloid labeling in the temporal region of the $\mathrm{AD}$ brain (Figure $3 \mathrm{C}$ ). Both the frontal and temporal cortices demonstrated C. pneumoniae intra- and extracellular immunoreactivity with multiple monoclonal antibodies (Figure 3B, D; Table 1, \#5, \#3, respectively). Both amyloid and chlamydial immunoreactivity were visible at low magnification. Interestingly, although C. pneumoniae was found in the frontal cortex in the AD cases, we observed more consistent evidence of $C$. pneumoniae in the temporal cortex. Further, Chlamydial immunoreactivity occurred in apposition with amyloid pathology in chlamydia positive areas. There was approximately $5-10 \%$ of the area immunolabeled with amyloid compared to $1-5 \%$ of the same area immunolabeled for intracellular and extracellular chlamydia. There appeared to be no consistent pattern to the area or region in which the immunolabeling occured. Furthermore, in the temporal cortex for 2 of 5 non-AD cases, C. pneumoniae labeling was observed, although less prominently as compared to the AD brains. These non AD cases also demonstrated diffuse amyloid immunopositivity (data not shown).

\section{Association of $C$. pneumoniae labeling and Thioflavin $S$ staining}

To further illustrate the relationship between amyloid and chlamydia, temporal AD sections were labeled with an anti-C. pneumoniae antibody (Table 1, \#5) (red) and then stained for Thioflavin $S$ (yellow-green fluorescence using the FITC filter ) on the same section. For this dual procedure, optimal results were obtained when immunohistochemistry (IHC) was performed prior to 

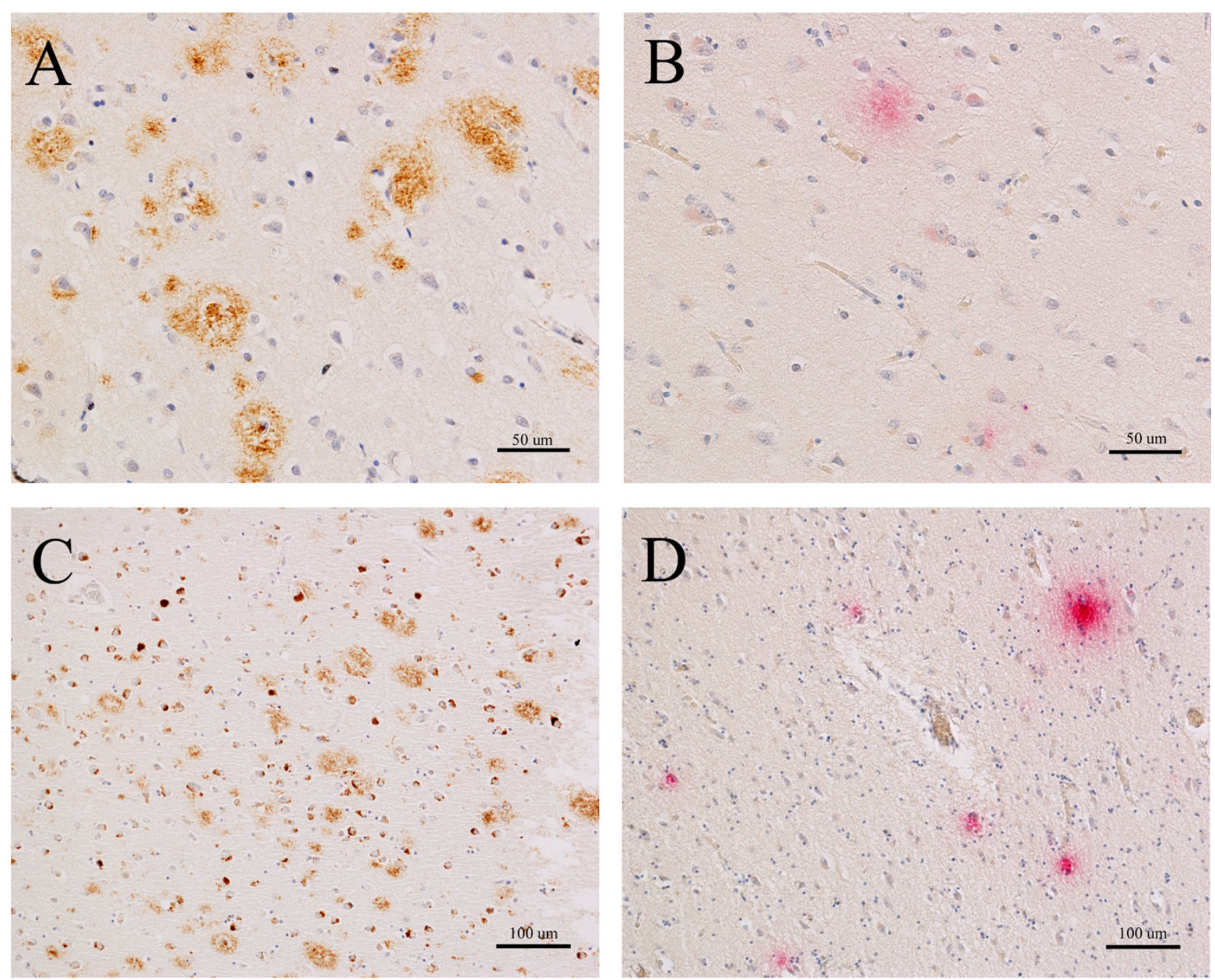

Figure 3 Amyloid and Chlamydia pneumoniae immunolabeling in AD tissue. Representative amyloid and C. pneumoniae immunolabeling detected in frontal (A, B) and temporal (C, D) sections of an AD brain. For amyloid labeling (brown color), two different anti-amyloid antibodies were used, (Sigma, panel A) and (Signet, panel C). Representative amyloid immunoreactivity, both extracellular plaques, including dense core plaques (A), and intracellular labeling (C) are revealed at a low magnification in order to appreciate the level of amyloid pathology. Likewise, C. pneumoniae immunoreactivity (magenta color) is evident at low magnification in both brain regions using two different monoclonal antibodies (Table 1, \#5 panel B), and (Table 1, \#3 panel D). Size bar A, B = 50 $\mu \mathrm{m} ; \mathrm{C}, \mathrm{D}=100 \mu \mathrm{m}$.

incubating with Thioflavin S. Both light and fluorescent images of the areas of interest were captured and then overlaid or merged. This approach revealed the close proximity of chlamydia with NFTs and NSPs, which are hallmarks of Alzheimer's disease pathology (Figure 4).

\section{Pre-absorption of anti-C. pneumoniae antibodies with Amyloid $\beta$ 1-40 and 1-42 peptides}

Upon detection of atypical extracellular chlamydia immunoreactivity, we questioned whether anti-chlamydia antibodies could label extracellular deposits of amyloid. To address this issue, we labeled several AD brain serial sections with separate anti-C. pneumoniae antibodies that had been pre-absorbed with amyloid peptides. The immunoreactivity for C. pneumoniae was not depleted after labeling with these pre-absorbed antibodies (Figure 5A-D).
Figure 5A demonstrated the representative extracellular and intracellular immunoreactivity of a non- pre-absorbed monoclonal anti-C. pneumoniae antibody (Table 1, \#3) on frontal AD tissue. Similar extracellular and intracellular immunolabeling of C. pneumoniae antigen was obtained with the antibody pre-absorbed against $>10$ molar excess Sigma amyloid $A \beta 1-40$ peptide (Figure $5 B$ ) and with the antibody pre-absorbed against $>10$ molar excess Sigma amyloid $A \beta 1-42$ peptide (Figure $5 C$, D). Comparable results were obtained with two other anti-C. pneumoniae antibodies pre-absorbed in the same manner (Table $1, \# 1$, \#2) (data not shown).

\section{Discussion}

Intracellular and extracellular C. pneumoniae immunoreactivity was observed in the entorhinal cortex, the 

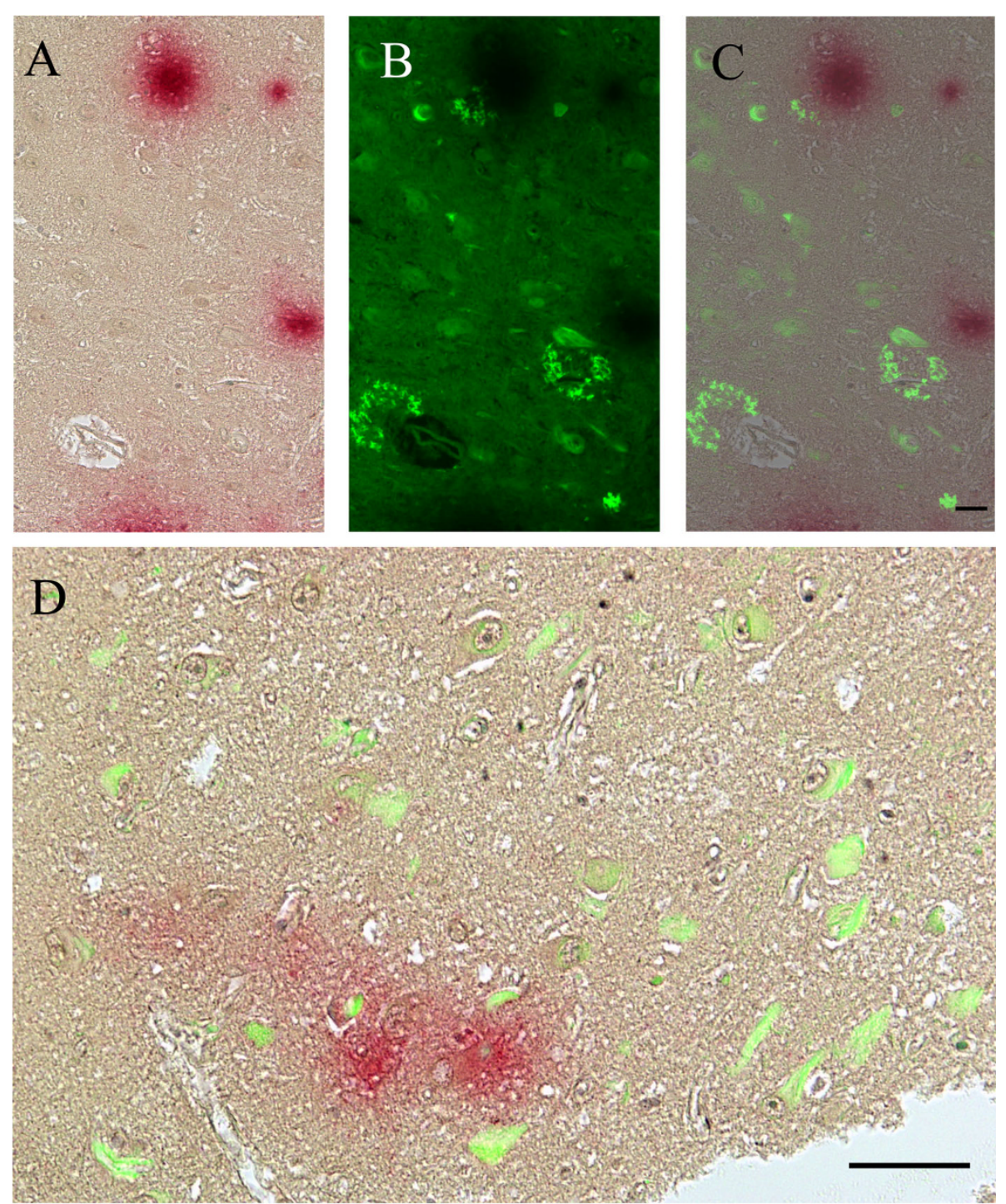

Figure 4 Dual Chlamydia pneumoniae labeling and Thioflavin S staining of the temporal cortex from an AD brain .Alzheimer's disease temporal cortex is immunolabeled with a monoclonal anti-C. pneumoniae antibody (Table 1, \#5) followed by staining with Thioflavin $\mathbf{S}$ on the same tissue specimen. Panel A shows intracellular and extracellular C. pneumoniae immunoreactivity (magenta color). Using a FITC filter, panel B shows both Thioflavin S positive intracellular labeling, presumably NFTs, and extracellular amyloid plaques (yellow fluorescence). Panel $C$ shows the light (panel A) (C. pneumoniae) image overlaid on the fluorescent (panel B) (Thioflavin S) image. Panel D shows merged light and fluorescent images of another region of this temporal cortex illustrating merged chlamydia chromogen immunoreactivity and Thioflavin $\mathrm{S}$ amyloid/tau fluorescence. Size bars $=50 \mu \mathrm{m}$.

hippocampal formation, and the frontal cortex, regions of the brain that typically demonstrate AD pathology. Clear discrimination between chlamydia immunoreactivity and age-related lipofuscin accumulation within neurons was demonstrated. Serial sections of brain tissue displayed both amyloid pathology and the presence of C. pneumoniae immunoreactivity. Thioflavin S staining for fibrillar amyloid and specific antibody labeling for C. pneumoniae revealed deposition of both when performed on the same section. As some C. pneumoniae labeling was extracellular, a more atypical pattern, pre-absorption studies with Amyloid $\beta$ 1-40 and 1-42 were performed. These studies revealed that C. pneumoniae antibodies were not cross-reacting with $\mathrm{A} \beta$. Collectively, these data demonstrate that evidence of C. pneumoniae infection is present in brain tissues in areas of amyloid pathology, thereby suggesting that an interrelationship exists between these entities in the pathogenesis of sporadic late-onset AD.

Immunolabeling for Chlamydia may be overlooked in brain tissues, as it is different from what is observed in cellular infections in vitro. In all AD samples analyzed 


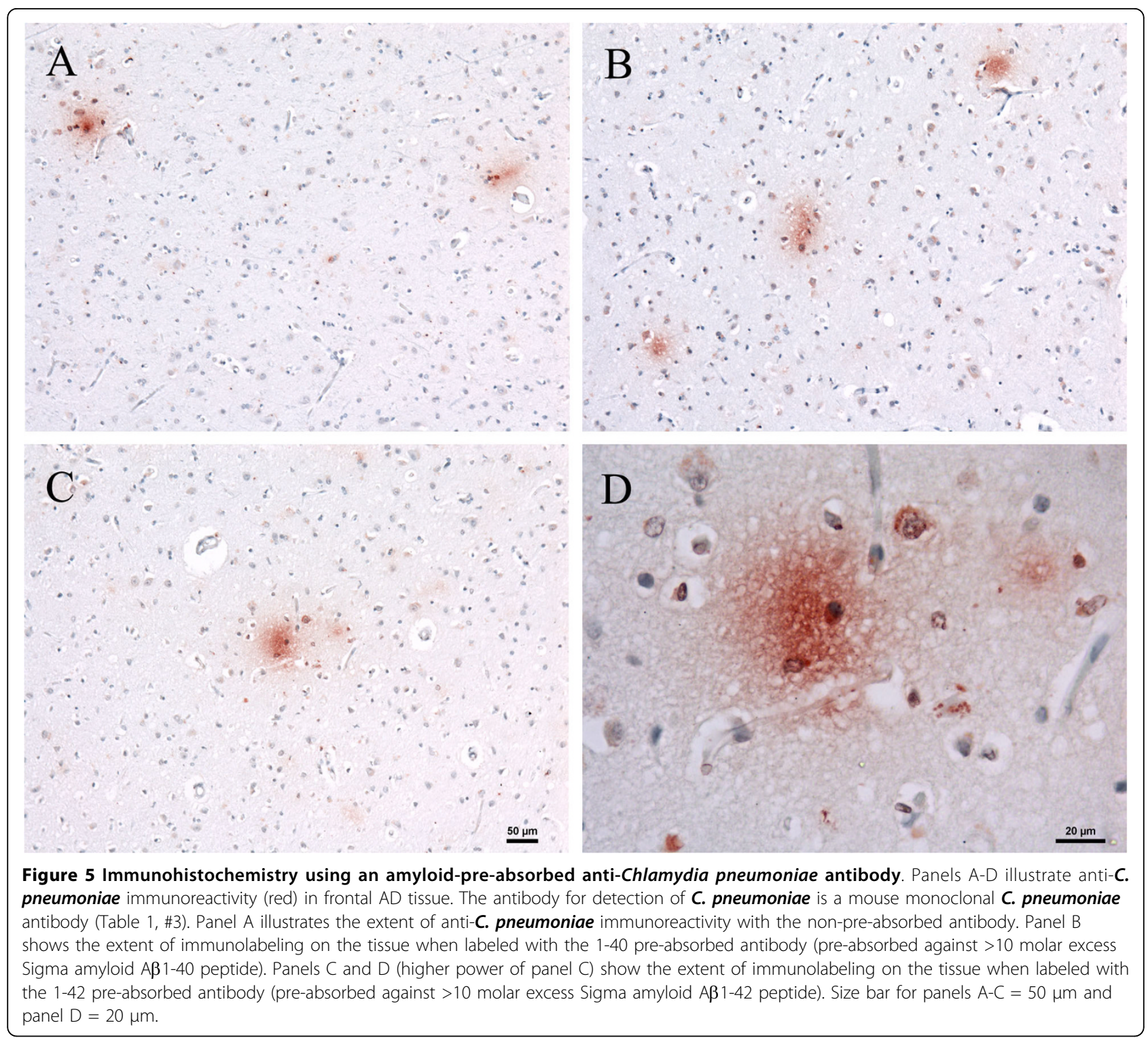

in this study, both typical intracellular perinuclear chlamydia immunoreactivity and atypical extracellular labeling were observed. Intracellular labeling demonstrated punctate elementary bodies and membrane bound inclusions similar to that of in vitro studies (see Figure 2E). This specific labeling was differentiated from lipofuscin by using red chromogens, either alkaline phosphatase (AP)red or AP magenta, as the substrate to denote $C$. pneumoniae immunoreactivity. Horseradish peroxidase labeling with 3, 3'-Diaminobenzidine (DAB), a brown chromogen, was not used as this labeling may be confused with the golden brown lipofuscin found in neurons of aged brains.

Two distinct extracellular patterns of chlamydia immunoreactivity were observed: one, a punctate pattern signifying the elementary body form of the bacteria, which can be extruded from infected cells into the surrounding milieu [31], and two, an amorphous foci pattern most likely indicating secreted chlamydial factors such as lipopolysaccharide [30,31]. These patterns in the cerebrum will require further study although similar profiles of Chlamydia labeling in situ have been demonstrated in a different organ [32]. Furthermore, our data demonstrated that C. pneumoniae extracellular immunoreactivity was not reflective of cross-reactivity with extracellular Amyloid $\beta$ 1-40 or 1-42. However, C. pneumoniae extracellular organism and related antigens may interact with extracellular proteins and lipids in the brain. Although not always in direct overlap with amyloid plaque deposits, chlamydial antigens may interact with soluble oligomeric forms of amyloid, such as ADDLs, that are less likely to be found in mature 
plaques due to their soluble nature [7]. These intriguing findings and their implications require further understanding of the possible relationship between amyloid and chlamydia in the same cortical regions of the brain. This relationship will vary with each individual AD patient. Each AD patient has different levels of pathology and may have corresponding variability in extent and distribution of C. pneumoniae infection in the cerebrum. Following further studies into this variability, the relationship between pathology and infection can be more thoroughly evaluated.

Although C. pneumoniae is principally a respiratory pathogen, infection of the brain has been shown following intranasal and lung infection $[33,34]$. In this regard, monocytes infected with $C$. pneumoniae in the lungs may spread the infection via the peripheral circulation to the brain through the blood brain barrier or circumventricular organs [22,23]. Alternatively, a more direct and insidious route of infection may follow the olfactory pathways. As such, the infection becomes established in the olfactory nasal neuroepithelia, progresses to the olfactory bulbs, and eventually infects brain structures such as the entorhinal cortex and hippocampus. The olfactory structures, the entorhinal cortex, and the hippocampal formation are the most vulnerable and the earliest regions affected in the onset of AD [35,36]. Our current study highlights $C$. pneumoniae detection in the frontal and temporal cortices, including the entorhinal cortex and the hippocampal formation. Thus, infection of these regions in the brain may have great impact on the development of AD pathology.

Previous studies have demonstrated C. pneumoniae in both human and animal olfactory bulbs $[8,33,34]$. In both cell culture and animal studies, C. pneumoniae has been shown to infect nasal neuroepithelial cells [34]. In the animal studies, infection appeared to spread centrifugally from the vulnerable neuronal cells in the olfactory bulb into the brain [33,34,37]. Further, Chlamydia isolated from AD brains in a prior study was shown to have more commonalities with Chlamydia respiratory strains than with Chlamydia strains from atherosclerosis with the suggestion that the organism itself may have a tropism for specific cell types in the CNS [38]. Upon consideration of these data and our current data, a rationale for the selective vulnerability of specific brain regions to infection and resultant pathology emerges.

Notably, histopathological amyloid plaques and tangles are used to define the stage of $\mathrm{AD}$, but the correlation with the pathology of the disease and the clinical manifestations of the disease are not always clear [2]. Some individuals who have massive pathology have little to no symptoms. On the other hand, some symptomatic individuals may show little pathology upon post-mortem histopathological examination. As such, there are many variations in the amount and type of damage evident in $\mathrm{AD}$ [1]. The variability of correlation between the symptomology and histopathology suggests other events and/or ingredients may be missing in the pathobiology of $\mathrm{AD}$.

The response in the brain to infection may determine the extent of pathology and symptomatology that may arise. In this regard, C. pneumoniae infection characteristically promotes an inflammatory response whereby cytokines such as IL- $1 \beta$ and TNF- $\alpha$ are secreted and may initiate cellular damage [39]. These cytokine responses to infection parallel similar responses to amyloid accumulation [40]. Additionally, another cellular response to $C$. pneumoniae infection in culture is the production and processing of amyloid peptides. Labeling of infected cells in culture for Amyloid $\beta$ 1-42 often reveals intracellular immunoreactivity at early postinfection times (unpublished observations CJH, DMA, CSL, BJB). Interestingly, our earlier study demonstrated intracellular and extracellular amyloid deposits in the brains of non-transgenic BALB/c mice following intranasal C. pneumoniae infection [34]. Our current study showed a similar relationship in which we demonstrated both amyloid and chlamydia immunoreactivity in the temporal cortex of the AD brain.

Further evaluation is required to specifically demonstrate how amyloid and C. pneumoniae, both intracellular and extracellular, are interrelated. In addition, future studies are required to further characterize the atypical extracellular Chlamydial immunolabeling profiles. Others have demonstrated evidence for an association of infection and amyloid in AD [41]. HSV1 viral DNA was shown to specifically associate with AD amyloid plaques [41]. Previous hypotheses have even suggested that amyloid in the AD brain may act as an antiviral agent [42] or an entrapping agent for infection [43]. Intriguingly, a recent study suggests that amyloid has anti-microbial properties, and may arise in response to brain infection in AD [44]. As 2 of 5 non-AD cases in our current study showed occasional chlamydia immunoreactivity and diffuse amyloid deposition, future analysis must also include mild cognitive impairment cases, as well as nonAD cases, as infection may be a prodromal event leading to eventual AD pathology.

\section{Conclusions}

The exact consequences of infection that correlate best with the stage and subtype of Alzheimer's disease require ongoing investigation. This is true especially with regard to the hallmarks of the disease such as amyloid plaques and tau tangles. Alzheimer's disease is manifest with multifactorial aspects of pathology and with potentially multiple associated environmental factors, including infectious agents [8,10-12]. Further, our 
Chlamydia pneumoniae data suggest that the areas of the brain involved with olfaction are important to study as infection in these regions, as well as in the olfactory neuroepithelia and olfactory bulbs, may be a precursor to the pathology associated with AD. Identification and localization of infectious agents, such as Chlamydia pneumoniae, in specifically vulnerable areas and cell types in the brain is paramount. This determination may be a missing link in the current strategy of associating symptomatology to disease pathogenesis in sporadic late-onset Alzheimer's disease.

\section{Methods}

\section{Human Post-mortem Samples}

Post-mortem tissue samples from various brain regions (frontal and temporal cortices) of patients with and without AD were obtained through Dr. William Hill of the Medical College of Georgia (Augusta, GA), from the MCP-Hahnemann School of Medicine Department of Pathology, currently Drexel University College of Medicine (Philadelphia, PA), and the Alzheimer's Research Center of the Health Partners Research Foundation at Regions Hospital (St Paul, MN) under approved protocols at each procurement site. Five AD and 5 non-AD age-matched male and female archival brains were examined. All cases were confirmed as AD or non-AD by neuropathological examination at the source using standard diagnostic criteria (NINDS/CERAD) [45].

\section{Optimization of Immunohistochemistry}

To detect chlamydia in the brain, IHC was performed on serial sections from formalin-fixed, paraffin-embedded Alzheimer brain tissue. Parallel IHC was performed using antibodies for AD amyloid plaque pathology, specifically anti-amyloid 1-42 antibodies. Amyloid plaques were best visualized when antigen retrieval was not performed whereas the optimal labeling for chlamydia antigens was achieved following antigen retrieval. Optimum immunoreactivity occurred at $37^{\circ} \mathrm{C}$ in a humidified chamber for both amyloid and chlamydial antigens. To visualize amyloid deposits, 3, 3' Diaminobenzidine (DAB) (golden brown color) was used. Red chromogens such as alkaline phosphatase (AP) red and AP new magenta were found to best visualize both intracellular and extracellular C. pneumoniae, as nothing in a typical brain section should appear red. For counterstaining, Mayer's hematoxylin was chosen as it only stains the nucleus leaving the cytoplasm of the cell clear allowing easier visualization of intracellular chlamydia EB and inclusion immunolabeling. Controls were used to rule out non-specific labeling. Our controls included: no primary or secondary antibodies, primary with no corresponding secondaries, no primary but HRP conjugated secondary and/or AP conjugated secondary alone and in combination, and anti-human IgG primary with appropriate HRP and/or AP secondaries alone and in combination, all of which were reacted with appropriate substrates DAB and/or AP red and/or AP magenta, alone and in combination. Controls for fluorescent work also included no primary, secondary, or stain to allow for evaluation of autofluorescence of the brain tissues.

\section{Chlamydia pneumoniae Immunohistochemistry}

Archival, paraffin-embedded, human brain tissues were deparaffinized through xylenes and graded alcohols. Antigen retrieval was performed in The Retriever (Electron Microscopy Science, Fort Washington, PA) according to manufacturer's directions using $1 \times$ Citra Antigen retrieval buffer (BioGenex, San Ramen, CA). The sections were rinsed with filtered water $3 \times 5$ min and treated with Alkaline Phosphatase/Horseradish Peroxidase Block (BioFX Laboratories, Owings Mills, $\mathrm{MD})$ for $30 \mathrm{~min}$ at room temperature (RT). The tissues were rinsed with filtered water, PBS $3 \times 5 \mathrm{~min}$, and blocked in $1 \%$ Fetal Bovine Serum (FBS)/PBS for $5 \mathrm{~min}$ at RT. Sections were incubated in a humidified chamber with anti-chlamydia primary antibodies for $90 \mathrm{~min}$ at $37^{\circ} \mathrm{C}$. Anti-chlamydial antibodies, genus and species specific, were purchased from commercial sources and used at dilutions shown in Table 1. Following the incubation, the sections were rinsed with PBS $3 \times 5$ min and blocked with 1\% FBS/PBS block for $5 \mathrm{~min}$ at $\mathrm{RT}$. The sections were incubated in a humidified chamber with appropriate anti-mouse or anti-rabbit Alkaline Phosphatase conjugated secondary antibody for $60 \mathrm{~min}$ at $37^{\circ} \mathrm{C}$ (BioFX Laboratories, Owings Mills, MD or Zymed- Invitrogen Corporation, Carlsbad, CA). After $3 \times 5$ min water rinses, the sections were reacted with BioFX AP-New Magenta IHC Substrate (BioFX Laboratories- SurModics, Owings Mills, MD) $40 \mathrm{~min}$ at $\mathrm{RT}$ or they were reacted with AP red (Zymed- Invitrogen Corporation, Carlsbad, CA) according to the manufacturer's directions. The sections were rinsed with water and counterstained with Mayer's hematoxylin (Electron Microscopy Science, Fort Washington, PA). The slides were aqueous mounted using Crystal Mount (Biomeda, Thermo Fisher Scientific, Pittsburgh, PA) and then permanently mounted using Permount (Thermo Fisher Scientific, Pittsburgh, PA). Sections were viewed on a Nikon Eclipse E800 or a Nikon Eclipse 90i microscope and captured using: the spot RT (Diagnostic Instruments, Starling Heights, MI) with the Image Pro Plus Phase 3 Imaging software (Media Cybernetics, Silver Spring, MD), the Nikon DS-Fi1 camera using the NIS-Elements Advanced Research version 3.0 software (Nikon) or the Nikon DS-Ri1 camera using the NIS-Elements Advanced Research version 3.0 software (Nikon). 
Depending on the available tissue, the IHC was performed on 2-3 serial sections for each antibody for each section of the brain that was available. Out of the battery of antibodies, 5 monoclonal and 1 polyclonal antibodies were more routinely used (Table 1 ). In order to be considered a positive sample, the sample must have been positive by IHC with at least 2 C. pneumoniae species-specific monoclonal antibodies in 2 of the 3 serial sections of each brain section. All chlamydia antibodies used on the tissue sections were also used on astrocyte, epithelial, monocyte, and/or neuronal cell lines that were uninfected or that had been infected with AR39 Chlamydia pneumoniae (ATCC, Manassas, VA) as antibody specificity controls.

\section{Amyloid Immunohistochemistry}

Archival, paraffin-embedded, human brain tissues were deparaffinized through xylenes and graded alcohols. The sections were rinsed with filtered water and phosphate buffered saline (PBS) $3 \times 5 \mathrm{~min}$. Endogenous peroxidase was quenched using $3 \% \mathrm{H}_{2} \mathrm{O}_{2}$ or Alkaline Phosphatase/ Horseradish Peroxidase Block (BioFX Laboratories, Owings Mills, MD) for $30 \mathrm{~min}$ at RT. The tissues were rinsed with sterile filtered water, PBS $3 \times 5 \mathrm{~min}$, and blocked in $1 \%(\mathrm{FBS}) / \mathrm{PBS}$ for $5 \mathrm{~min}$ at RT. The primary antibody, mouse anti-human $\beta$-Amyloid (4G8, Signet, Covance, Cambridge, MA) or rabbit anti-Amyloid peptide $\beta$ cleavage site 42 (Sigma, St Louis, MO), were incubated in a humidified chamber for $90 \mathrm{~min}$ at $37^{\circ} \mathrm{C}$. Following the incubation, the sections were rinsed with PBS $3 \times 5$ min and blocked in 1\% FBS/PBS block for 5 $\mathrm{min}$ at RT. The sections were incubated with anti-rabbit horseradish peroxidase conjugated (HRP) secondary antibody (Zymed, Invitrogen Corporation, Carlsbad, CA) in a humidified chamber for $60 \mathrm{~min}$ at $37^{\circ} \mathrm{C}$. After a water rinse and PBS washes, $3 \times 5 \mathrm{~min}$, the sections were reacted with 3, 3'-Diaminobenzidine (DAB) (Sigma Aldrich, St Louis MI) for 10-20 min at RT. The sections were rinsed with water and counterstained with Mayer's hematoxylin (Electron Microscopy Science, Fort Washington, PA). Slides were dehydrated through graded alcohols and xylenes followed by cover slipping using Permount (Thermo Fisher Scientific, Pittsburgh, PA). Sections were viewed and captured as noted previously.

\section{Chlamydia pneumoniae antibody pre-absorption}

Individual anti-chlamydia antibodies, mouse antiC. pneumoniae (Table 1, \#2, \#3, BioDesign, Meridian Life Science, Inc., Saco, ME) or rabbit anti-chlamydia antibody (Table 1, \#1, BioDesign, Meridian Life Science, Inc., Saco, ME), were mixed with $>10 \mathrm{M}$ excess of either Amyloid $\beta$ 1-40 or Amyloid $\beta$ 1-42 peptide (Sigma, St Louis, MO) in a batch method and allowed to incubate overnight at $4^{\circ} \mathrm{C}$. The supernatant of each preabsorbed antibody was then used for Chlamydia pneumoniae immunohistochemistry as described above with the exception that secondary antibodies were conjugated with HRP and reacted with DAB.

\section{Dual Chlamydia pneumoniae Immunohistochemistry and Thioflavin S staining}

Slides immunolabeled for C. pneumoniae were stained using a modified Thioflavin $\mathrm{S}$ protocol http://wwwmedlib.med.utah.edu/WebPath/webpath.html[46]. In brief, following Chlamydia pneumoniae primary and subsequent secondary incubations and AP-New Magenta substrate development, slides were counterstained with Mayer's hematoxylin and coversliped with aqueous mount. Areas of chlamydia immunoreactivity were identified by light microscopy. The aqueous mount was then soaked off and the section were immersed in $1 \%$ Thioflavin S for $5 \mathrm{~min}$, differentiated in $70 \%$ alcohol for $5 \mathrm{~min}$, and coverslipped with water. The sections were kept wet by cover slipping with water as the intensity of the Thioflavin S was diminished with use of Crystal mount. Sections were viewed and captured while still wet using a Nikon Eclipse E800 microscope and Nikon DS-Ri1 camera. Light and fluorescent (under FITC filter) images were captured and merged using the NISElements Advanced Research version 3.0 software (Nikon).

\section{Quantitative analysis}

For determination of amyloid and chlamydia labeling and the relationship between the two in the sections, we evaluated the relationship with the following quantification schemes.

For percentage of chlamydia pneumoniae immunopositiyity and percentage of type of immunopositivity, we first qualitatively determined the types of label to be counted: intracellular, extracellular or a combination of the two. In representative frontal and temporal sections, the total numbers of positives across a section were counted and then the numbers of each category tallied and the percentage of the total positive immunoreactivity was determined per total area across the section. Analysis at 4, 10 and 40× magnification was performed manually and utilizing the NIS-Elements Advanced Research version 3.0 software (Nikon). Using the software, the immunoreactivity was selected and the area or cells were counted. A similar quantification scheme was used in determining the relationship of the chlamydia immunoreactivity relative to the amyloid immunoreactivity across a representative temporal region. In this case, we again qualitatively determined the type of immunoreactivity: intracellular, extracellular, or a mixture of the two for amyloid and chlamydia. We then 
determined the counts and areas of single label and then the label of both in apposition to get an indication of the relative relationships between the two antigens

\section{Acknowledgements}

We are grateful to Dr. William Hill, the Alzheimer's Research Center of the Health Partners Research Foundation at Regions Hospital and the autopsy service of Medical College of Pennsylvania/Hahnemann University (Drexel University) for tissues provided for this work. We are grateful to the families who donated tissues to the several sources listed above for research into Alzheimer's disease. We would like to thank Gwendolyn Harley for all her sectioning help. Finally, we would like to thank all the students past and present that helped with the work including Adia Taylor, and Jesty Abraham.

\section{Author details}

'Pathology/Microbiology/Immunology and Forensic Medicine Department, Philadelphia College of Osteopathic Medicine, 4170 City Ave, Philadelphia, Pennsylvania, USA. ${ }^{2}$ Neuroscience/Physiology/Pharmacology Department, Philadelphia College of Osteopathic Medicine, 4170 City Ave, Philadelphia, Pennsylvania, USA. ${ }^{3}$ Center for Chronic Disorders of Aging, Philadelphia College of Osteopathic Medicine, 4170 City Ave, Philadelphia, Pennsylvania, USA.

\section{Authors' contributions}

CJH participated in design and coordination of experiments, prepared tissue samples, carried out immunohistochemistry experiments, analysis and drafted the manuscript.

LRH participated in immunohistochemistry experiments and helped to draft the manuscript.

RJH participated in immunohistochemistry experiments, quantitative analysis and data compilation.

CSL participated in design and pre-absorbed Chlamydia antibodies with amyloid peptides.

DMA participated in design and helped prepare tissue samples

BJB conceived of the study, participated in design and analysis, prepared tissue samples, and helped draft the manuscript.

All authors read and approved of the final manuscript.

Received: 1 April 2010 Accepted: 23 September 2010

Published: 23 September 2010

\section{References}

1. Duyckaerts C, Delatour B, Potier MC: Classification and basic pathology of Alzheimer disease. Acta Neuropathol 2009, 118(1):5-36

2. Savva GM, Wharton $S B$, Ince PG, Forster G, Matthews FE, Brayne C, Medical Research Council Cognitive Function and Ageing Study: Age neuropathology, and dementia. N Engl I Med 2009, 360(22):2302-2309.

3. Gouras GK, Almeida CG, Takahashi RH: Intraneuronal Abeta accumulation and origin of plaques in Alzheimer's disease. Neurobiol Aging 2005, 26(9):1235-1244

4. D'Andrea MR, Nagele RG, Wang HY, Lee DH: Consistent immunohistochemical detection of intracellular beta-amyloid42 in pyramidal neurons of Alzheimer's disease entorhinal cortex. Neurosci Lett 2002, 333(322317315):163-16.

5. Takahashi RH, Milner TA, Li F, Nam EE, Edgar MA, Yamaguchi H, Beal MF, $\mathrm{Xu}$ H, Greengard P, Gouras GK: Intraneuronal Alzheimer abeta42 accumulates in multivesicular bodies and is associated with synaptic pathology. Am J Pathol 2002, 161(5):1869-1879.

6. Friedrich RP, Tepper K, Rönicke R, Soom M, Westermann M, Reymann K, Kaether C, Fändrich M: Mechanism of amyloid plaque formation suggests an intracellular basis of $\mathrm{A} \beta$ pathogenicity. Proceedings of the National Academy of Sciences 2010, 107(5):1942-1947.

7. Lambert MP, Barlow AK, Chromy BA, Edwards C, Freed R, Liosatos M, Morgan TE, Rozovsky I, Trommer B, Viola KL, Wals P, Zhang C, Finch CE, Krafft GA, Klein WL: Diffusible, nonfibrillar ligands derived from Abeta1-42 are potent central nervous system neurotoxins. Proc Natl Acad Sci USA 1998, 95(11):6448-6453.

8. Balin BJ, Gerard HC, Arking EJ, Appelt DM, Branigan PJ, Abrams JT, Whittum-Hudson JA, Hudson AP: Identification and localization of
Chlamydia pneumoniae in the Alzheimer's brain. Med Microbiol Immunol (Berl) 1998, 187(1):23-42.

9. Miklossy J: Alzheimer's disease-a spirochetosis? Neuroreport 1993, 4(7):841-88.

10. Miklossy J, Khalili K, Gern L, Ericson RL, Darekar P, Bolle L, Hurlimann J, Paster BJ: Borrelia burgdorferi persists in the brain in chronic lyme neuroborreliosis and may be associated with Alzheimer disease. $J$ Alzheimers Dis 2004, 6(6):639-49, discussion 673-81.

11. Itzhaki RF, Lin WR, Shang D, Wilcock GK, Faragher B, Jamieson GA: Herpes simplex virus type 1 in brain and risk of Alzheimer's disease [see comments]. Lancet 1997, 349(904797167222):241-244.

12. Gerard HC, Dreses-Werringloer U, Wildt KS, Deka S, Oszust C, Balin BJ, Frey WH, Bordayo EZ, Whittum-Hudson JA, Hudson AP: Chlamydophila (Chlamydia) pneumoniae in the Alzheimer's brain. FEMS Immunol Med Microbiol 2006, 48(3):355-366.

13. Michelow IC, Olsen K, Lozano J, Rollins NK, Duffy LB, Ziegler T, Kauppila J, Leinonen M, McCracken GH Jr: Epidemiology and clinical characteristics of community-acquired pneumonia in hospitalized children. Pediatrics 2004, 113(4):701-77.

14. Gieffers J, Pohl D, Treib J, Dittmann R, Stephan C, Klotz K, Hanefeld F, Solbach W, Haass A, Maass M: Presence of Chlamydia pneumoniae DNA in the cerebral spinal fluid is a common phenomenon in a variety of neurological diseases and not restricted to multiple sclerosis. Ann Neurol 2001, 49(5):585-589.

15. Campbell LA, Kuo CC: Chlamydia pneumoniae and atherosclerosis. Semin Respir Infect 2003, 18(1):48-54.

16. Vikatmaa $\mathrm{P}$, Lajunen $\mathrm{T}$, Ikonen $T S$, Pussinen $\mathrm{PJ}$, Lepantalo $\mathrm{M}$, Leinonen $\mathrm{M}$, Saikku P: Chlamydial lipopolysaccharide (cLPS) is present in atherosclerotic and aneurysmal arterial wall-cLPS levels depend on disease manifestation. Cardiovasc Pathol 2010, 19(1):48-54

17. Bandaru VC, Laxmi V, Neeraja M, Alladi S, Meena AK, Borgohain R, Keerthi AS, Kaul S: Chlamydia pneumoniae antibodies in various subtypes of ischemic stroke in Indian patients. J Neurol Sci 2008, 272(1-2):115-122.

18. Du C, Yao SY, Ljunggren-Rose A, Sriram S: Chlamydia pneumoniae infection of the central nervous system worsens experimental allergic encephalitis. J Exp Med 2002, 196(12):1639-1644.

19. Sriram S, Ljunggren-Rose A, Yao SY, Whetsell WO Jr: Detection of chlamydial bodies and antigens in the central nervous system of patients with multiple sclerosis. J Infect Dis 2005, 192(7):1219-1228.

20. Appelt DM, Roupas MR, Way DS, Bell MG, Albert EV, Hammond CJ, Balin BJ: Inhibition of apoptosis in neuronal cells infected with Chlamydophila (Chlamydia) pneumoniae. BMC Neurosci 2008, 9:13.

21. Boelen E, Steinbusch HW, van der Ven AJ, Grauls G, Bruggeman CA Stassen FR: Chlamydia pneumoniae infection of brain cells: an in vitro study. Neurobiol Aging 2007, 28(4):524-532.

22. Maclntyre A, Abramov R, Hammond CJ, Hudson AP, Arking EJ, Little CS, Appelt DM, Balin BJ: Chlamydia pneumoniae infection promotes the transmigration of monocytes through human brain endothelial cells. $J$ Neurosci Res 2003, 71(5):740-750.

23. Maclntyre A, Hammond CJ, Little CS, Appelt DM, Balin BJ: Chlamydia pneumoniae infection alters the junctional complex proteins of human brain microvascular endothelial cells. FEMS Microbiol Lett 2002, 217(2):167-172.

24. Mahony JB: Chlamydiae host cell interactions revealed using DNA microarrays. Ann N Y Acad Sci 2002, 975:192-201.

25. Zhong $G$, Fan $P$, Ji H, Dong $F$, Huang $Y$ : Identification of a chlamydial protease-like activity factor responsible for the degradation of host transcription factors. J Exp Med 2001, 193(8):935-942.

26. Pollack DV, Croteau NL, Stuart ES: Uptake and intra-inclusion accumulation of exogenous immunoglobulin by Chlamydia-infected cells. BMC Microbiol 2008, 8:213.

27. Fischer SF, Hacker G: Characterization of antiapoptotic activities of Chlamydia pneumoniae in infected cells. Ann N Y Acad Sci 2003, 1010:565-567.

28. Fischer SF, Vier J, Kirschnek S, Klos A, Hess S, Ying S, Hacker G: Chlamydia inhibit host cell apoptosis by degradation of proapoptotic BH3-only proteins. J Exp Med 2004, 200(7):905-916.

29. van Zandbergen G, Gieffers J, Kothe H, Rupp J, Bollinger A, Aga E, Klinger M, Brade H, Dalhoff K, Maass M, Solbach W, Laskay T: Chlamydia pneumoniae multiply in neutrophil granulocytes and delay their spontaneous apoptosis. J Immunol 2004, 172(3):1768-1776. 
30. Stuart ES, Troidle KM, MacDonald AB: Chlamydial glycolipid antigen: Extracellular accumulaton, biological activity, and antibody recognition. 1994, 28(2):85-90.

31. Hybiske K, Stephens RS: Mechanisms of host cell exit by the intracellular bacterium Chlamydia. Proc Natl Acad Sci USA 2007, 104(27):11430-11435.

32. Fernandez-Obregon A, Patton DL: The role of Chlamydia pneumoniae in the etiology of acne rosacea: response to the use of oral azithromycin Cutis 2007, 79(2):163-167.

33. Little CS, Bowe A, Lin R, Litsky J, Fogel RM, Balin BJ, Fresa-Dillon KL: Age alterations in extent and severity of experimental intranasal infection with Chlamydophila pneumoniae in BALB/c mice. Infect Immun 2005, 73(3):1723-1734

34. Little CS, Hammond CJ, MacIntyre A, Balin BJ, Appelt DM: Chlamydia pneumoniae induces Alzheimer-like amyloid plaques in brains of BALB/C mice. Neurobiol Aging 2004, 25(4):419-429.

35. Christen-Zaech S, Kraftsik R, Pillevuit O, Kiraly M, Martins R, Khalili K, Miklossy J: Early olfactory involvement in Alzheimer's disease. Can J Neurol Sci 2003, 30(1):20-25.

36. Mann DM, Tucker CM, Yates PO: Alzheimer's disease: an olfactory connection? Mech Ageing Dev 1988, 42(1):1-15.

37. Itzhaki RF, Wozniak MA, Appelt DM, Balin BJ: Infiltration of the brain by pathogens causes Alzheimer's disease. Neurobiol Aging 2004, 25(5):619-627.

38. Dreses-Werringloer U, Bhuiyan M, Zhao Y, Gerard HC, Whittum-Hudson JA, Hudson AP: Initial characterization of Chlamydophila (Chlamydia) pneumoniae cultured from the late-onset Alzheimer brain. Int J Med Microbiol 2009, 299(3):187-201.

39. Rasmussen SJ, Eckmann L, Quayle AJ, Shen L, Zhang YX, Anderson DJ, Fierer J, Stephens RS, Kagnoff MF: Secretion of proinflammatory cytokines by epithelial cells in response to Chlamydia infection suggests a central role for epithelial cells in chlamydial pathogenesis. J Clin Invest 1997, 99(197148774):77-87.

40. Lue LF, Brachova L, Civin WH, Rogers J: Inflammation, A beta deposition, and neurofibrillary tangle formation as correlates of Alzheimer's disease neurodegeneration. J Neuropathol Exp Neurol 1996, 55(1097010972):1083-108

41. Wozniak MA, Mee AP, Itzhaki RF: Herpes simplex virus type 1 DNA is located within Alzheimer's disease amyloid plaques. J Pathol 2009, 217(1):131-138.

42. Kammerman EM, Neumann DM, Ball MJ, Lukiw W, Hill JM: Senile plaques in Alzheimer's diseased brains: possible association of beta-amyloid with herpes simplex virus type 1 (HSV-1) L-particles. Med Hypotheses 2006, 66(2):294-299.

43. Bishop GM, Robinson SR: Physiological roles of amyloid-beta and implications for its removal in Alzheimer's disease. Drugs Aging 2004, 21(10):621-630.

44. Soscia SJ, Kirby JE, Washicosky KJ, Tucker SM, Ingelsson M, Hyman B, Burton MA, Goldstein LE, Duong S, Tanzi RE, Moir RD: The Alzheimer's Disease-Associated Amyloid beta-Protein Is an Antimicrobial Peptide. PloS One 2010, 5(3):e9505.

45. Mirra SS, Heyman A, McKeel D, Sumi SM, Crain BJ, Brownlee LM, Vogel FS, Hughes JP, van Belle G, Berg L: The Consortium to Establish a Registry for Alzheimer's Disease (CERAD). Part II. Standardization of the neuropathologic assessment of Alzheimer's disease. Neurology 1991, 41(491187203):479-486.

46. WebPath: Internet Pathology Laboratory. [http://library.med.utah.edu/ WebPath/HISTHTML/MANUALS/THIOFLAV.PDF].

doi:10.1186/1471-2202-11-121

Cite this article as: Hammond et al:: Immunohistological detection of Chlamydia pneumoniae in the Alzheimer's disease brain. BMC

Neuroscience 2010 11:121.

\section{Submit your next manuscript to BioMed Central and take full advantage of:}

- Convenient online submission

- Thorough peer review

- No space constraints or color figure charges

- Immediate publication on acceptance

- Inclusion in PubMed, CAS, Scopus and Google Scholar

- Research which is freely available for redistribution

Submit your manuscript at www.biomedcentral.com/submit
Ciomed Central 